\title{
Análise espacial dos aglomerados de nascimentos ocorridos em hospitais SUS e não SUS do município de São Paulo, Brasil
}

\author{
Spatial analysis of live birth clusters in Unified Health System (SUS) \\ and private hospitals in the municipality of São Paulo, Brazil
}

Patrícia Carla dos Santos ${ }^{1}$

Zilda Pereira da Silva ${ }^{1}$

Francisco Chiaravalloti Neto ${ }^{1}$

Marcia Furquim de Almeida ${ }^{1}$

${ }^{1}$ Faculdade de Saúde Pública, Universidade de São Paulo. R. Brigadeiro Galvão 540, Barra Funda. 01.151-000 São Paulo SP. patcarla@usp.br
Abstract The scope of this study is to evaluate the spatial distribution of live births (LB) in the Municipality of São Paulo (MSP), verify if there is spatial dependence, identify possible differences in birth profiles and evaluate the distances between homes and delivery hospitals. LB occurring in high complexity hospitals were studied, namely 4 from the Unified Health System (SUS) and 4 from the private network in MSP in 2008. 46,190 LB were geocoded: 48.8\% from SUS hospitals and $51.2 \%$ from private hospitals, accounting for $27.9 \%$ of total live births in MSP. LB in SUS hospitals formed two clusters, with a high proportion of households with incomes of $1 / 2$ to $2 M W$ and a marked number of shantytowns and high birth rates. $L B$ in private hospitals formed a cluster in the central region of MSP, where there is a high proportion of households with $>10 \mathrm{MW}$ income and a low birth rate. Differences in maternal, pregnancy and childbirth characteristics were statistically significant in SUS and non-SUS hospitals, as well as the frequency of multiple births. There was no difference in the prevalence of low birth weight and pre-term births. The results showed differences in the LB cluster profile reflecting the current inequalities in living conditions.

Key words Live births, Spatial analysis, Geographic distance, Health inequalities, Unified Health System (SUS) hospitals, Private hospitals
Resumo O objetivo deste estudo é avaliar a distribuição espacial de nascidos vivos (NV) do município de São Paulo (MSP), verificar se há dependência espacial, identificar possíveis diferenças no perfil dos nascimentos e avaliar as distâncias percorridas entre os domicílios e os hospitais do parto. Foram estudados os NV ocorridos em hospitais de alta complexidade, quatro SUS e quatro da rede não SUS no MSP em 2008. Foram georreferenciados 46.190 NV: 48,8\% em hospitais SUS e 51,2\% não SUS, estes representaram 27,9\% do total de NV do MSP. Os NV de hospitais SUS formaram dois aglomerados com elevada proporção de domicílios com renda de 1/2 a 2SM, concentração de favelas e altas taxas de natalidade. Os NV de hospitais não SUS formaram um aglomerado na região central do MSP, onde há elevada proporção de domicílios com renda > 10 SM e baixa natalidade. Foram encontradas diferenças estaticamente significantes das características maternas, da gestação e do parto entre os NV de hospitais SUS e não SUS e da frequência de gemelaridade. Não houve diferença na prevalência de baixo peso e pré-termo. Os resultados mostraram existir diferenciais no perfil dos aglomerados de NV, que refletem as desigualdades das condições de vida do MSP.

Palavras-chave Nascidos vivos, Análise espacial, Deslocamento geográfico, Desigualdades em saúde, Hospitais SUS, Hospitais privados 


\section{Introdução}

São Paulo tornou-se uma megacidade segundo critério das Nações Unidas ao custo de um crescimento desordenado e, consequentemente, sem infraestrutura adequada para atender todas as necessidades da população. Além disso, tal desordem se expressa na ocupação do espaço urbano. A cidade de São Paulo foi o polo econômico hegemônico do crescimento econômico brasileiro na segunda fase de industrialização do país, recebendo elevado contingente migratório, nas décadas de 1960 e 1970. O processo de urbanização no país se deu por meio da metropolização, com a formação das áreas periféricas das cidades $^{1}$. Essas áreas se caracterizavam por infraestrutura urbana precária e déficit de equipamentos sociais, entre os quais os de saúde, com elevada concentração de moradias subnormais ${ }^{2}$. O acesso à saúde é resultado da composição social do espaço e está relacionado à disponibilidade geográfica dos equipamentos de saúde e da forma como a rede de serviços se organiza ${ }^{3}$. Diferentes condições de vida resultam em situações de saúde desiguais ${ }^{4}$, entre as quais perfis epidemiológicos de nascimentos. Assim, entende-se o espaço como um campo de forças cuja aceleração é desigual ${ }^{5}$ e não apenas como uma dimensão do meio ambiente e externo aos indivíduos.

A ocorrência de partos em hospitais é influenciada por fatores socioeconômicos, pela infraestrutura de prestação de serviços e por políticas públicas assistenciais e preventivas 6 . A rede hospitalar brasileira é heterogênea, tanto em termos de distribuição espacial quanto a outras características (tipo de prestador, porte, infraestrutura e complexidade dos serviços oferecidos), incluindo a rede de assistência obstétrica ${ }^{7}$. No município de São Paulo, segundo a pesquisa Assistência Médico Sanitária (MAS) ${ }^{8}$, a participação dos leitos em estabelecimentos privados com internação no país em 2009 era de 60,6\% sendo que destes $45,1 \%$ tinham vínculo com o SUS.

A expansão do SUS na década de 1990, com a garantia de assistência gratuita ao parto, teve papel fundamental nas elevadas taxas de partos hospitalares ${ }^{7}$. No município de São Paulo, a atenção ao parto é predominantemente hospitalar, com 99,4\% dos nascimentos no ano de 2008 tendo ocorridos em hospitais. Segundo dados do estudo conduzido com dados de $2006^{6}$, a rede SUS respondeu por 57,6\% dos nascimentos hospitalares no município de São Paulo (MSP). A atenção ao parto no MSP nesse ano concentrouse em hospitais de alta complexidade, sendo
$55,1 \%$ dos nascimentos SUS e 30,4\% não SUS ocorreram em hospitais de alta complexidade.

Segundo dados da ANS, a taxa de cobertura por plano de saúde no MSP em 2011 era de 59,0\%, assim o acesso de parcela significativa das gestantes paulistanas se dá por meio da utilização de planos de saúde e o fluxo delas irá depender das condições socioeconômicas, da oferta de serviços, da livre escolha e da localização dos hospitais que prestam este tipo de atenção.

A definição do fluxo 'mãe - hospital do parto' não depende apenas da organização dos sistemas de saúde municipais e estaduais. De acordo com as diretrizes do Programa de Humanização no Pré-Natal e Nascimento (PHPN) do Ministério da Saúde, a orientação da gestante sobre a unidade hospitalar SUS a ser procurada no momento do parto é de responsabilidade dos profissionais que a assistem no pré-natal ${ }^{9}$.

A Secretaria Municipal de Saúde do município de São Paulo criou em 2006 o programa Rede de Proteção à Mãe Paulistana (RPMP), com ações voltadas para garantir o acesso da gestante e do recém-nascido em todas as unidades de saúde do SUS, desde a atenção básica até a alta complexidade. Com isso há vinculação da gestante com o hospital do parto, conforme orientação do Ministério da Saúde.

As técnicas de georrefereciamento permitem visualizar as relações complexas que ocorrem na ocupação do solo urbano ${ }^{10}$ e trouxeram um avanço aos estudos de diferenciais intraurbanos em saúde ${ }^{10-16}$, pois permitem a observação de diferenciais de condições de vida e saúde, que não seriam captados ao se trabalhar com unidades de referência de maiores dimensões, como os distritos administrativos onde pode ocorrer homogeneização de condições de vida diferenciadas ${ }^{17-20}$.

O objetivo deste estudo é avaliar a distribuição espacial de nascimentos ocorridos em oito hospitais de alta complexidade do MSP (rede SUS e não SUS) por meio do georreferenciamento dos endereços de residência materna e hospitais, verificar se há formação de aglomerados de nascidos vivos (NV) e identificar possíveis diferenças no perfil das mães e recém-nascidos desses aglomerados.

\section{Material e método}

Trata-se de um estudo ecológico cuja unidade de análise é o conjunto de nascimentos de mães residentes no município de SP ocorridos no período de 01/01/2008 a 31/12/2008 em oito hospitais 
de alta complexidade, sendo quatro da rede SUS e quatro da rede não SUS.

Foram utilizadas as informações das Declarações de Nascido Vivo (DNV) obtidas do banco de dados unificado SEADE/SES (Fundação SEADE/Secretaria de Estado da Saúde de São Paulo). Informações dos hospitais foram obtidas a partir do Cadastro Nacional de Estabelecimentos de Saúde (CNES), do Ministério da Saúde.

Para caracterizar os aglomerados de nascimentos foram utilizados dados de renda familiar e domicílios subnormais do Censo Demográfico 2010 (IBGE); a taxa de natalidade e fecundidade (Fundação SEADE); leitos hospitalares SUS e não SUS por mil habitantes e; taxa de Unidades Básicas de Saúde (UBS) por 20 mil habitantes, segundo distrito administrativo do MSP (SMS/ SP). Também foi utilizada base Cartográfica de Favelas (CEM/CEBRAP).

Os hospitais da amostra foram selecionados levando-se em consideração o volume de partos no ano de 2008, vínculo com o SUS e a entidade mantenedora (Municipal, Organização Social de Saúde - OSS, Filantrópico e Estadual). Os oito hospitais estudados são considerados como de alta complexidade na atenção ao parto e ao recém-nascido, seguindo o critério de Silva et al. ${ }^{21}$. Nestes hospitais ocorreram 48.429 nascimentos.

O georreferenciamento consiste em atribuir coordenadas geográficas a cada registro do banco de dados, por meio de operações realizadas num ambiente de Sistema de Informações Geográficas (SIG). Os endereços são vinculados a um sistema de coordenadas geográficas (expressas em latitude e longitude) através da base de logradouros. Para o georreferenciamento dos endereços foram utilizadas a base geográfica digitalizada dos distritos administrativos do município de São Paulo e a base de logradouros, disponibilizadas pelo Centro de Estudos da Metrópole (CEM/CEBRAP). Foram realizadas a verificação de completitude e a padronização dos endereços de residência materna e dos hospitais para obtenção das coordenadas.

Dos 48.429 registros foram excluídos 637 $(1,3 \%)$ devido à ausência de registro concomitante da rua e CEP. Dos 47.792 endereços 20.857 estavam com todos os campos preenchidos (rua, número e CEP), os 26.935 endereços incompletos foram complementados com base nas informações da Empresa Brasileira de Correios e Telégrafos - Correios. Não foi possível recuperar a informação e georreferenciar 1.602, endereços (764 de NV SUS e 838 NV não SUS). Os 46.190 endereços que permaneceram no estudo foram padronizados e georreferenciados utilizando-se a base de logradouros do MSP, dos quais 22.557 eram de NV ocorridos em hospitais SUS e 23.633 de NV em hospitais não SUS.

A partir das coordenadas foram calculadas as distâncias (em linha reta) da residência materna ao hospital de nascimento, obtendo-se média, mediana e percentis das distâncias para cada hospital. Os aglomerados de nascimento foram obtidos utilizando-se a técnica Kernel, através do aplicativo gratuito TerraView 4.1.0, que fornece a densidade de eventos por $\mathrm{km}^{2}$. Para definir o raio dos aglomerados foi utilizada a maior mediana de distância entre o hospital e a residência materna.

O projeto foi submetido e aprovado pelo Comitê de Ética em Pesquisa da Faculdade de Saúde Pública - USP, conforme Resolução no 196/ $96^{22}$ da Comissão Nacional de Pesquisa.

\section{Resultados}

Foram georreferenciados $46.190 \mathrm{NV}$ endereços de residência materna, sendo $22.557(48,8 \%)$ de hospitais SUS e $23.633(51,2 \%)$ não SUS. Estes nascimentos representam $27,9 \%$ do total de nascimentos hospitalares de mães residentes no MSP em 2008.

A distância média percorrida pela parturiente de sua residência ao hospital do parto foi de $9,6 \mathrm{~km}$. Para os hospitais SUS a média de distância até a residência da mãe foi de $9,2 \mathrm{~km}$, e não SUS a distância média foi de $9,9 \mathrm{~km}$ (Tabela 1). A mediana de distância $(7,9 \mathrm{~km})$ percorrida pelas mães dos nascidos nos hospitais SUS é menor que a mediana de distância dos não SUS $(9,4 \mathrm{~km})$, não havendo diferença de distâncias a partir do percentil 75. Quatro hospitais possuem média de distância maior que a média total (1 e 2, SUS; 5 e 8 , não SUS).

Os hospitais 3 e 4 apresentaram as menores médias de distância percorrida pelas mães $(4,1$ $\mathrm{km}$ e $4,4 \mathrm{~km}$ ) e o percentil 90 situa-se abaixo da média total (Tabela 1).

Com base no valor mais elevado da mediana de distância encontrado (Hospital $1=12,4 \mathrm{~km}$ ), foi determinado o raio de $12,5 \mathrm{~km}$ para determinação dos aglomerados dos 8 hospitais e para todos os nascimentos SUS e não SUS (Figuras 1 a 3 ).

$A$ área quente representa a maior densidade de $\mathrm{NV} / \mathrm{km}^{2}$, esta área do Hospital SUS 1 compreende os distritos de Campo Grande, Santo Amaro, Socorro, Cidade Dutra, Jardim São Luís, Campo Belo, Saúde, Cursino, Jabaquara, Cidade Ademar e Pedreira. O conjunto destes distri- 
Tabela 1. Distância (em km) da residência materna ao hospital de nascimento, segundo vínculo e hospital.

\begin{tabular}{|c|c|c|c|c|c|c|c|c|c|c|}
\hline \multirow{2}{*}{ Vínculo/Hospital } & \multirow{2}{*}{$\mathbf{n}$} & \multirow{2}{*}{ Média } & \multirow{2}{*}{ DP } & \multirow{2}{*}{ Min } & \multirow{2}{*}{$\operatorname{Max}$} & \multicolumn{5}{|c|}{ Percentis } \\
\hline & & & & & & 10 & 25 & 50 & 75 & 90 \\
\hline SUS & 22.557 & 9,2 & 6,7 & 0,0 & 46,1 & 2,0 & 3,5 & 7,9 & 13,2 & 18,5 \\
\hline Hospital 1 & 8.227 & 12,8 & 6,7 & 0,1 & 40,3 & 4,9 & 7,6 & 12,4 & 16,9 & 21,9 \\
\hline Hospital 2 & 5.863 & 11,2 & 4,6 & 0,6 & 43,7 & 6,1 & 7,6 & 10,6 & 13,5 & 17,6 \\
\hline Hospital 3 & 5.558 & 4,4 & 4,8 & 0,0 & 46,1 & 1,3 & 2,0 & 2,9 & 4,7 & 8,8 \\
\hline Hospital 4 & 2.909 & 4,1 & 4,3 & 0,1 & 37,4 & 1,2 & 1,9 & 3,2 & 4,4 & 7,8 \\
\hline Não-SUS & 23.633 & 9,9 & 5,5 & 0,1 & 35,9 & 3,2 & 5,8 & 9,4 & 13,0 & 17,5 \\
\hline Hospital 5 & 8.353 & 12,3 & 5,7 & 0,2 & 35,9 & 4,8 & 8,4 & 12,0 & 16,0 & 20,2 \\
\hline Hospital 6 & 6.282 & 8,3 & 4,6 & 0,1 & 32,4 & 2,8 & 5,0 & 7,6 & 11,1 & 14,1 \\
\hline Hospital 7 & 5.815 & 8,3 & 4,8 & 0,1 & 35,9 & 2,5 & 4,6 & 8,1 & 10,9 & 14,1 \\
\hline Hospital 8 & 3.183 & 9,8 & 5,4 & 0,1 & 35,5 & 3,2 & 5,8 & 9,6 & 12,7 & 16,8 \\
\hline Total & 46.190 & 9,6 & 6,1 & 0,0 & 46,1 & 2,4 & 4,7 & 8,8 & 13,1 & 17,9 \\
\hline
\end{tabular}

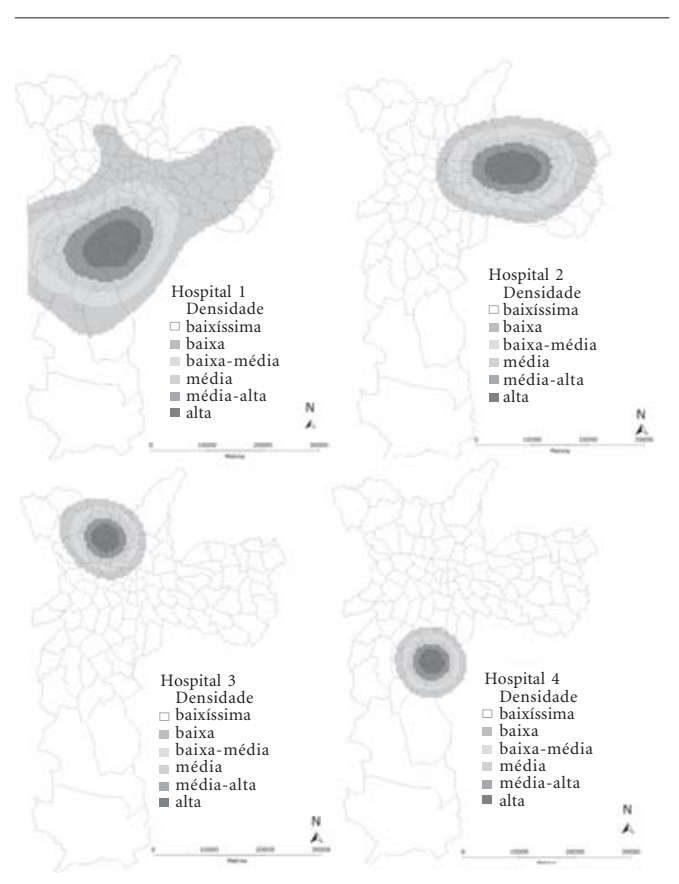

Figura 1. Densidade de nascimentos em hospitais SUS segundo local de residência materna, por hospital de nascimento. MSP, 2008.

tos apresentou uma taxa média de natalidade de 15,2\%o habitantes e possuía também 0,68 UBS por 20 mil habitantes, em 2008 e 21,4\% dos domicílios desta área o chefe tinha renda de até 2 Salários Mínimos (SM), segundo dados do censo demográfico em 2010. Para o Hospital SUS 2, a área quente compreende os distritos de Penha, Cangaíba, Vila Matilde, Carrão, Tatuapé, Vila

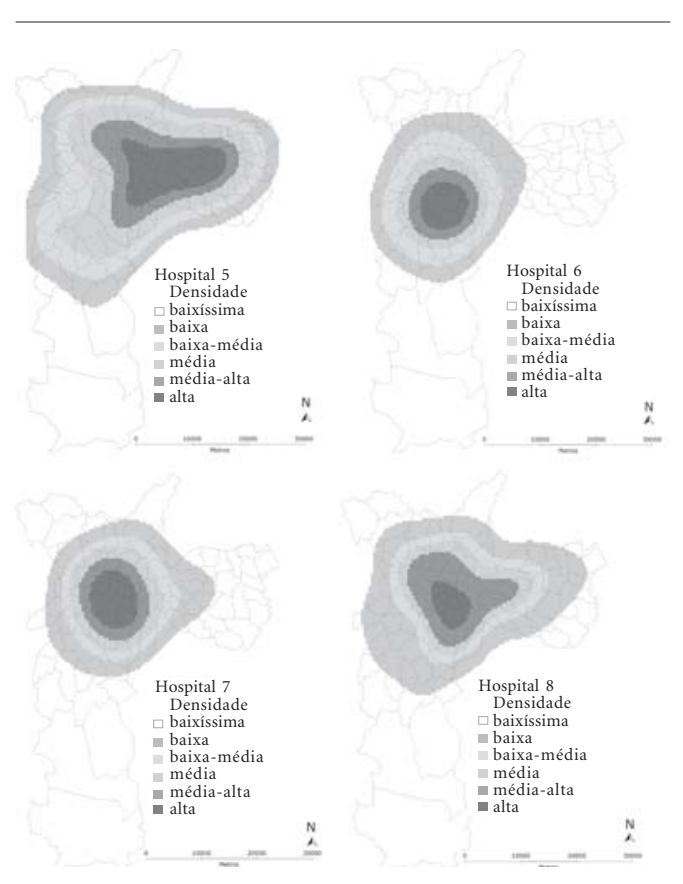

Figura 2. Densidade de nascimentos em hospitais não SUS segundo local de residência materna, por hospital de nascimento. MSP, 2008.

Maria, Belém, Moóca, Água Rasa, Vila Formosa, Aricanduva, Cidade Líder, Artur Alvim e Ponte Rasa. $\mathrm{Na}$ área formada por esses distritos a taxa média de natalidade era de 13,9\%o habitantes e 19,8\% dos domicílios de baixa renda e 0,72 UBS por 20 mil habitantes. A área de maior densidade de NV do Hospital SUS 3 tem como os distritos de Brasilândia, Cachoeirinha, Mandaqui, 

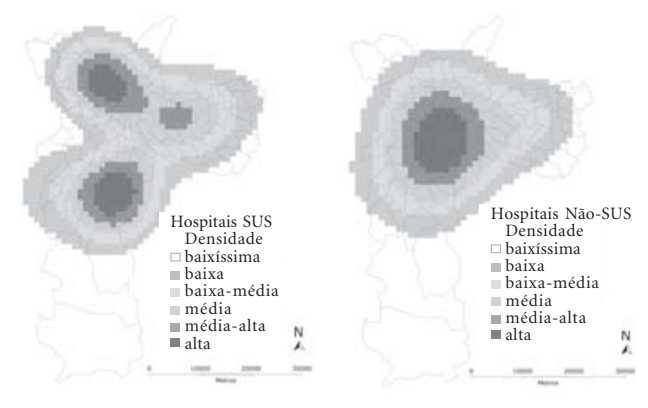

Figura 3. Densidade de nascimentos em hospitais SUS e não SUS segundo local de residência materna. MSP, 2008.

Casa Verde, Limão, Freguesia do Ó e Pirituba. Nesta área a taxa média de natalidade era de 16,8\%o habitantes e $23,4 \%$ domicílios de baixa renda a taxa de UBS por 20.000 habitantes era de 0,75 e do Hospital SUS 4 os distritos de Jabaquara, Cidade Ademar, Pedreira, Campo Grande e Cidade Dutra Nesta área a taxa de natalidade era de $16,2 \%$ o habitantes e a proporção de domicílios com renda inferior a $2 \mathrm{SM}$ era de $24,9 \%$ e a taxa de UBS era 0,65 .

A área de maior concentração de NV do Hospital não SUS 5 abrange os distritos da região Centro-Leste do MSP Santa Cecília, Bela Vista, Consolação, Sé, Liberdade, Vila Mariana, Cursino, Ipiranga, Vila Prudente, Moóca, Cambuci, Brás, Pari, Belém, Casa Verde, Santana, Vila Guilherme, Vila Maria, Penha, Ponte Rasa, Artur Alvim, Cidade Líder, Aricanduva, São Lucas, Água Rasa, Tatuapé, Vila Formosa, Carrão e Vila Matilde. A taxa de natalidade desta área era 13,3\%o habitantes e a proporção de domicílios com baixa renda era de renda 15,9\%. O Hospital não SUS 6 tem sua área quente a região que abrange os distritos de Morumbi, Itaim Bibi, Moema, Jardim Paulista, Pinheiros, Butantã, Vila Sônia, Vila Andrade, Santo Amaro, Campo Belo, Saúde e Vila Mariana a natalidade desta área era de $12,2 \%$ o habitantes e proporção de domicílios de baixa renda era de 8,6\% (Figura 3).

As áreas de abrangência dos hospitais não SUS 7 e 8 são semelhantes e compreendem os distritos de Barra Funda, Perdizes, Pinheiros, Itaim Bibi, Moema, Vila Mariana, Ipiranga, Moóca, Pari, Bom Retiro, Santa Cecília, Consolação, Bela Vista, Liberdade, Cambuci, Brás, Sé e República a natalidade desta área era de $12,3 \%$ o NV e a proporção de domicílios de baixa renda era de 9,2\% (Figura 3).
Os nascimentos ocorridos em hospitais não SUS são em maioria de mães residentes nos distritos de Jabaquara, Sacomã, Ipiranga, Saúde, Cursino, Vila Mariana, Campo Belo, Moema, Itaim Bibi, Jardim Paulista, Morumbi, Vila Andrade, Lapa, Pinheiros, Butantã, Alto de Pinheiros, Perdizes, Barra Funda, Bom Retiro, Santa Cecília, República, Consolação, Cambuci, Liberdade, Bela Vista, Sé, Pari, Santana, Vila Maria, Vila Guilherme, Limão, Casa Verde, Vila Prudente, Belém, Tatuapé, Água Rasa, Moóca e Brás.

Para o total de NV ocorridos em hospitais SUS observa-se a formação de duas áreas quentes principais, uma ao Sul e outra ao Norte, com ramificação para a região Leste. Essas áreas quentes englobam quase a totalidade dos nascimentos dos hospitais 3 e 4, pois o percentil 90 da distância até a residência materna até o hospital fica abaixo dos $12,5 \mathrm{~km}$.

As áreas quentes dos aglomerados de nascimentos em hospitais SUS estão localizadas em distritos com elevada proporção de domicílios com renda de $1 / 2$ a 2 SM, maior concentração de favelas e cortiços e altas taxas de natalidade.

Os aglomerados de nascimentos em hospitais não SUS formaram aglomerados semelhantes entre si situados nos distritos da região Central do MSP, com elevada proporção de domicílios com renda superior a $10 \mathrm{SM}$, com menores taxas de natalidade e baixas taxas de UBS por 20 mil habitantes.

Encontraram-se diferenças estatisticamente significantes entre as características maternas, da gestação e do parto dos nascimentos em hospitais SUS e não SUS, exceto para a prevalência de baixo peso ao nascer e para nascimentos prétermo. A gemelaridade foi duas vezes maior entre os nascimentos não SUS (4,3\%) que do SUS $(2,1 \%)$. A frequência de mães adolescentes foi praticamente 18 vezes maior nos nascimentos em hospitais SUS $(21,5 \%)$ do que no não SUS (1,2\%), enquanto que a proporção de mães com mais de 35 anos foi 3,2 vezes maior entre os nascimentos em hospitais não SUS (30,0\%) que no SUS $(9,5 \%)$. A frequência de alta escolaridade materna (mais de 12 anos de estudo) foi 12,8 vezes maior nos nascimentos em hospitais não SUS $(83,3 \%)$ que entre as mães usuárias SUS (6,5\%) (Tabela 2).

Entre os nascimentos em hospitais não SUS, há uma proporção maior de mães primíparas $(63,7 \%)$ dos que nos hospitais SUS $(45,6 \%)$ e as grandes multíparas são mais 21 vezes mais frequentes entre os NV SUS (6,3\%) do que os do não SUS (0,3\%). A proporção de mulheres com 
Tabela 2. Perfil dos nascimentos de residentes nos aglomerados segundo características da mãe, do parto e do RN, ocorridos em hospitais SUS e não SUS. MSP, 2008.

\begin{tabular}{|c|c|c|c|c|c|c|c|}
\hline \multirow{2}{*}{ Características } & \multicolumn{2}{|c|}{ SUS } & \multicolumn{2}{|c|}{ Não-SUS } & \multicolumn{2}{|c|}{ Total } & \multirow[b]{2}{*}{ p } \\
\hline & $\mathrm{N}$ & $\%$ & $\mathbf{N}$ & $\%$ & $\mathbf{N}$ & $\%$ & \\
\hline \multicolumn{8}{|l|}{ Escolaridade da mãe } \\
\hline até 11 anos & 12.707 & 93,5 & 1.926 & 16,7 & 14.633 & 58,2 & $<0,001$ \\
\hline mais de 12 anos & 879 & 6,5 & 9.629 & 83,3 & 10.508 & 41,8 & \\
\hline \multicolumn{8}{|l|}{ Idade da mãe } \\
\hline menos de 19 anos & 2.930 & 21,5 & 134 & 1,2 & 3.064 & 12,2 & $<0,001$ \\
\hline de 20 a 34 anos & 9.410 & 69,0 & 7.974 & 68,9 & 17.384 & 69,0 & \\
\hline 35 ou mais & 1.291 & 9,5 & 3.473 & 30,0 & 4.764 & 18,9 & \\
\hline \multicolumn{8}{|l|}{ Paridade } \\
\hline Nenhum filho & 6.143 & 45,6 & 7.345 & 63,7 & 13.488 & 53,9 & $<0,001$ \\
\hline de 1 a 3 filhos & 6.485 & 48,1 & 4.150 & 36,0 & 10.635 & 42,5 & \\
\hline 4 ou mais filhos & 844 & 6,3 & 34 & 0,3 & 878 & 3,5 & \\
\hline \multicolumn{8}{|c|}{ Número de consultas pré natal } \\
\hline 0 a 3 & 968 & 7,1 & 21 & 0,2 & 989 & 3,9 & $<0,001$ \\
\hline 4 a 6 & 3.501 & 25,7 & 206 & 1,8 & 3.707 & 14,7 & \\
\hline 7 ou mais & 9.151 & 67,2 & 11.336 & 98 & 20.487 & 81,4 & \\
\hline \multicolumn{8}{|l|}{ Tipo de gravidez } \\
\hline Única & 13.343 & 97,9 & 11.080 & 95,7 & 24.423 & 96,9 & $<0,001$ \\
\hline Múltipla & 288 & 2,1 & 501 & 4,3 & 789 & 3,1 & \\
\hline \multicolumn{8}{|l|}{ Tipo de parto } \\
\hline Vaginal & 9.811 & 72,0 & 1.607 & 13,9 & 11.418 & 45,3 & $<0,001$ \\
\hline Cesáreo & 3.820 & 28,0 & 9.974 & 86,1 & 13.794 & 54,7 & \\
\hline \multicolumn{8}{|l|}{ Peso ao nascer } \\
\hline$<1.500 \mathrm{~g}$ & 214 & 1,6 & 156 & 1,3 & 370 & 1,5 & 0,195 \\
\hline $1500 \mathrm{~g}$ a $2.499 \mathrm{~g}$ & 1.104 & 8,1 & 898 & 7,8 & 2.002 & 7,9 & \\
\hline $2.500 \mathrm{~g}$ ou mais & 12.299 & 90,3 & 10.513 & 90,9 & 22.812 & 90,6 & \\
\hline \multicolumn{8}{|l|}{ Idade Gestacional } \\
\hline Pré-termo & 1.363 & 10,0 & 1.186 & 10,3 & 2.549 & 10,1 & 0,560 \\
\hline Termo & 12.222 & 90,0 & 10.378 & 89,7 & 22.600 & 89,9 & \\
\hline
\end{tabular}

menos de 7 consultas de pré-natal foi de 16.8 vezes mais frequente nas usuárias SUS $(32,8 \%)$ que nas usuárias não SUS (2,0\%). Com relação ao tipo de parto observou-se que as cesáreas foram 3,1 vezes mais frequentes entre as mães dos NV que formaram o aglomerado não SUS $(86,1 \%)$ que no aglomerado SUS (28\%) (Tabela 2).

\section{Discussão}

Neste estudo o georreferenciamento foi utilizado como instrumento analítico partindo da hipótese de que as informações individualizadas contidas no SINASC poderiam vir a formar agregados espaciais. Estes agregados não se constituem apenas na somatória dos indivíduos que os compõem, mas expressam a interação existente entre os indivíduos que formam estes agrupamentos, os quais compartilham entre si valores, comportamentos e características socioeconômicas ${ }^{17}$, entre as quais a utilização de serviços de saúde, e irão expressar a dimensão contextual do grupo, que neste estudo é representada pela dimensão social do espaço.

Os resultados obtidos revelam que houve a formação de aglomerados de nascimentos indicando existir uma associação espacial destes eventos. Estes resultados são concordantes com estudo realizado na $\mathrm{RMSP}^{23}$ e distintos daqueles obtidos em estudos realizados no Rio de Janeiro $^{16}$ e em Belo Horizonte ${ }^{16}$ onde não se encontrou dependência espacial dos NV. A localização dos aglomerados formados pelos nascimentos ocorridos em hospitais SUS e não SUS confirmaram os diferenciais intraurbanos já observados em outros estudos que utilizaram técnicas de georreferenciamento ${ }^{13-16,24,25}$. 
O cálculo das distâncias potenciais entre as residências maternas e os hospitais de nascimento não levou em conta o trajeto percorrido pela gestante, nem o tipo de transporte utilizado. Assim, os diferenciais que poderiam ser expressos através desse tipo de acesso encontram-se minimizados.

Verificou-se que as distâncias da residência materna ao hospital de parto são menores para os nascimentos ocorridos em hospitais SUS. Resultado semelhante foi obtido em estudo realizado com nascimentos ocorridos na RMSP no ano de $2004^{23}$ que mostrou que os hospitais públicos possuíam uma inserção mais regional para a atenção ao parto do que os hospitais privados.

As maiores médias de distâncias encontradas para mães usuárias do SUS dos hospitais 1 e 2 deve-se ao fato destes hospitais disporem de elevado número de leitos e destinarem-se exclusivamente à atenção ao parto e ao recém nascido. A opção de usar o maior valor de mediana das distâncias da residência até o hospital do parto fez com que houvesse a formação das áreas quentes do aglomerado de NV SUS nas regiões Sul e Norte do MSP, onde estão localizados os hospitais SUS 3 e 4, para os quais esta distância corresponde ao percentil 90. As menores distâncias potenciais percorridas pelas mães dos nascimentos ocorridos nestes hospitais indicam que há regionalização da atenção ao parto e atuação da atenção primária por meio do programa Mãe Paulistana.

As áreas quentes dos aglomerados de nascimentos em hospitais SUS estão localizadas em distritos com elevada proporção de domicílios com renda de 1/2 a 2 SM, maior concentração de favelas e cortiços, altas taxas de natalidade e com maior disponibilidade de atenção primária SUS, o que pode indicar que esta rede tende a promover a equidade da atenção primária em saúde.

A localização do aglomerado de NV não SUS em regiões com maior renda já era esperada, tendo em vista que os partos realizados em hospitais não SUS são pagos via desembolso direto ou por meio da cobertura de planos privados de saúde $^{26}$. O município de São Paulo tem elevada taxa de cobertura de saúde suplementar (59,0\%) o que facilita o acesso à rede privada não SUS.

Houve uma diferença significativa entre as proporções de mães com alta escolaridade nos aglomerados SUS e não SUS. Tal diferença também foi observada em estudo de georreferenciamento de nascimentos por setor censitário e IPVS (Índice Paulista de Vulnerabilidade Social) no $\mathrm{MSP}^{20}$ e outro realizado junto à maternidades públicas e privadas do Rio de Janeiro ${ }^{27}$. Segundo dados da PNDS-2006 (Pesquisa Nacional de Demografia e Saúde da Criança e da Mulher), o setor privado e o suplementar de saúde respondeu por quase $81 \%$ dos partos de mulheres com 12 ou mais anos de estudo, enquanto o SUS respondeu por pelo menos $86 \%$ dos partos de mulheres com menos de nove anos de estudo ${ }^{28}$, sendo a escolaridade um diferencial marcante entre a assistência pública ou privada no momento do parto.

As diferenças entre as proporções de mães adolescentes e com mais de 35 anos para mães de NV em hospitais SUS e não SUS foram significativamente expressivas. Dentre as mães com mais de 35 anos a diferença foi mais acentuada que aquela encontrada no estudo de Leal et al. ${ }^{27}$ e de Minuci e Almeida ${ }^{20}$. Estudo realizado no Município do Rio de Janeiro ${ }^{8}$ mostrou existir maior proporção de mães adolescentes em bairros com menor infraestrutura. Estudo realizado no Vale da Paraíba ${ }^{29}$ identificou existir correlação espacial negativa entre a proporção de mães adolescentes e o IDH (Índice de desenvolvimento $\mathrm{Hu}$ mano) dos municípios. Aglomerados de mães adolescentes também foram encontrados em áreas com piores condições socioeconômicas em Belo Horizonte ${ }^{16}$ e São Carlos (SP $)^{10}$.

$\mathrm{O}$ diferencial existente entre os aglomerados de nascimentos SUS e não SUS com relação à paridade materna se dá de forma mais acentuada entre as mães grandes multíparas. Diferença no perfil reprodutivo das mães também foi encontrada em estudo realizado em Belo Horizon$\mathrm{te}^{11}$. O resultado obtido apresentou diferencial mais acentuado que aquele observado em outros estudos ${ }^{19,27}$. É importante ressaltar que as áreas com elevada proporção de grandes multíparas coincidem com áreas de elevadas taxas de natalidade.

Observou-se elevada proporção de mães que realizaram 7 consultas ou mais de pré-natal. Esta proporção foi maior dentre as mães atendidas em hospitais não SUS. Outros autores ${ }^{28,30}$ verificaram existir relação desigual entre vínculo da atenção (SUS e não SUS) e número de consultas pré-natal. A diferença foi de 1,5 vezes entre as mães SUS e não SUS; note-se que esta diferença é menor que os diferenciais socioeconômicos observados. Este fato aliado à distribuição da rede básica de saúde, que se situa predominantemente na área periférica da cidade, sugere que a rede SUS atua no sentido de promover uma maior equidade no acesso à atenção pré-natal no MSP.

Foi encontrada elevada proporção de partos cesáreos no aglomerado de NV em hospitais não 
SUS, onde também há elevada proporção de mães com mais de 12 anos de estudo. Vários estudos têm mostrado existir uma associação entre a alta escolaridade e a realização de cesáreas ${ }^{11,31,32}$. Dados da PNDS-2006 revelaram que o parto cesáreo foi realizado em $78,8 \%$ das usuárias dos planos de saúde e que as taxas de cesárea são maiores quanto maior for a cobertura por plano de saúde $^{28}$. Estudos realizados com puérperas no Rio Grande do Sul ${ }^{24}$ e no Rio de Janeiro ${ }^{33}$ também mostraram alta proporção de partos cesáreos entre as usuárias privado $(69,8 \%$ e $88,1 \%$, respectivamente). Uma possível explicação para a alta prevalência de cesáreas na assistência não SUS é a possibilidade de escolha e da negociação entre o médico e a parturiente, que muitas vezes resulta em cesáreas eletivas pré-agendadas ${ }^{32,34}$.

As gestações múltiplas foram duas vezes mais frequentes entre as mães dos aglomerados não SUS do que no SUS. A elevada participação de mães com mais de 35 anos, com alta escolaridade e renda no aglomerado não SUS sugere que parte destes nascimentos se deve ao emprego de fertilização artificial, que devido ao seu elevado custo deve ser mais frequente neste grupo de nascimentos. Entretanto, o estudo realizado em Pe$\operatorname{lotas}^{34}$ não encontrou associação entre partos gemelares e renda.

A ausência de diferença da proporção de nascimentos pré-termo entre os aglomerados SUS e não SUS é semelhante aos resultados obtidos por Minuci e Almeida ${ }^{20}$ no MSP, que não encontraram resultados estatisticamente significantes nos aglomerados de nascimentos segundo IPVS. Entretanto, com relação ao baixo peso ao nascer essas autoras encontraram diferenciais intraurbanos para os nascimentos não pré-termo de baixo peso. A ausência de diferenças de baixo peso ao nascer possivelmente se deve ao fato de que entre os nascimentos não SUS há elevada partici- pação de mães com idade acima de 35 anos e nos nascimentos do aglomerado SUS há elevada participação de mães adolescentes e estes dois grupos etários são considerados como risco para nascimentos de baixo peso ${ }^{27,35}$. Em que pese ainda a análise de nascimentos ocorridos apenas em hospitais de alta complexidade dos sistemas público e privado do MSP.

Ao analisar apenas uma amostra dos nascimentos em hospitais de alta complexidade SUS e não SUS foram encontrados diferenciais socioeconômicos mais acentuados que aqueles existentes no universo dos usuários SUS e não SUS. Silva et al..$^{21}$ em estudo realizado na Região Metropolitana de São Paulo verificaram que a rede não SUS difere da rede SUS, mostrando que a atenção ao parto ofertada pela segunda era formada por $40 \%$ de hospitais de alta complexidade, enquanto que na primeira esta proporção era de $26,3 \%$. Esse estudo mostrou também que na rede não SUS à medida que diminuía a complexidade do hospital de nascimento aumentava a proporção de mães adolescentes e de baixa escolaridade e diminuía a proporção de mães de 35 anos e mais. Assim, ao se estudar apenas os nascimentos ocorridos em hospitais de alta complexidade não SUS superestima-se os diferenciais socioeconômicos existentes entre os usuários do sistema público e privado no MSP.

Em resumo, os dados deste estudo mostraram que existem diferenciais no perfil dos aglomerados de NV segundo o vínculo SUS e não SUS, que refletem os diferenciais intraurbanos existentes no MSP, entretanto estas diferenças não se expressaram em diferenciais expressivos das distâncias teóricas percorridas pelas mães entre as suas residências e hospitais de nascimento, sugerindo que a organização da assistência do SUS pode minimizar parte dos diferenciais sociais existentes.

\section{Colaboradores}

O artigo foi baseado na dissertação de mestrado de PC Santos, apresentada à Faculdade de Saúde Pública em 2012. ZP Silva, F Chiaravalloti Neto e MF Almeida participaram igualmente de todas as etapas de elaboração do artigo. 


\section{Referências}

1. Brito F. O deslocamento da população brasileira para as metrópoles. Estudos Avançados 2006; 20(57): 221-236.

2. Baltrusis N, D'ottaviano MCL. Ricos e pobres, cada qual em seu lugar: a desigualdade socio-espacial na metrópole paulistana. Cad. CRH 2009; 22(55):135149.

3. Travassos C, Oliveira EXG, Viacava F. Desigualdades geográficas e sociais no acesso aos serviços de saúde no Brasil: 1998 e 2003. Cien Saude Colet 2006; 11(4):975-986.

4. Hino P, Villa TCS, Cunha TN da, Santos CB dos. Padrões espaciais da tuberculose e sua associação à condição de vida no município de Ribeirão Preto. Cien Saude Colet 2011; 16(12):4795-4802.

5. Travassos C, Martins M. Uma revisão sobre os conceitos de acesso e utilização de serviços de saúde. Cad Saude Publica 2004; 20(2):S190-S198.

6. Santos M. A natureza do espaço: técnica e tempo, razão e emoção. São Paulo: Hucitec; 1996.

7. Silva ZP. Perfil dos nascimentos e da mortalidade neonatal precoce, segundo local de parto, complexidade hospitalar e rede SUS e não-SUS, região metropolitana de São Paulo, 2006 [tese]. São Paulo: Faculdade de Saúde Pública da USP; 2008.

8. Instituto Brasileiro de Geografia e Estatística (IBGE). Estatísticas de Saúde: Assistência Médico-Sanitária 2009. Rio de Janeiro: IBGE, 2010.

9. Brasil. Ministério da Saúde (MS). Secretaria de Políticas de Saúde. Programa de Humanização no Prénatal e Nascimento (PHPN). Brasília: MS; 2000.

10. Ferreira RA, Ferriani MGC, Mello DF, Carvalho IP, Cano MA, Oliveira LA. Análise espacial da vulnerabilidade social da gravidez na adolescência. Cad Saude Publica 2012; 28(2):313-323.

11. Friche AAL, Caiffa WT, Cesar CC, Goulart LMF, Almeida MCM. Indicadores de saúde materno infantil em Belo Horizonte, Minas Gerais, Brasil, 2001: análise dos diferenciais intra-urbanos. Cad Saude Publica 2006; 22(9):1955-1965.

12. Nogueira MJ, Silva BFA, Barcelos SM, Schall VT. Análise da distribuição espacial da gravidez adolescente no Município de Belo Horizonte - MG. Rev. bras. epidemiol 2009; 12(3):297-312.

13. Barcellos C, Acosta LMW Lisboa E, Bastos FI. Surveillance of mother-to-child HIV transmission: socioeconomic and health care coverage indicators. Rev Saude Publica 2009; 43(6):1006-1014.

14. Predebon KM, Mathias TAF, Aidar T, Rodrigues AL. Desigualdade sócio-espacial expressa por indicadores do Sistema de Informações sobre Nascidos Vivos (SINASC). Cad Saude Publica 2010; 26(8):15831594.

15. Melo EC, Mathias TAF. Spatial Distribution and Self-Correlation of Mother and Child Health Indicators in the State of Parana, Brazil. Rev. LatinoAm. Enfermagem 2010; 18(60):1177-1186.

16. D’Orsi E, Carvalho MS, Cruz OG. Similarity between neonatal profile and socioeconomic index: a spatial approach. Cad Saude Publica 2005; 21(3):786794.

17. Susser M, Susser E. Choosing a future for epidemiology I: Eras and paradigms. Am J Public Health 1996; 86(5):668-673.
18. Carvalho MS, Souza-Santos R. Análise de dados espaciais em saúde pública: métodos, problemas, perspectivas. Cad Saude Publica 2005; 21(2):361-378.

19. Morais Neto OL, Barros MBA, Martelli CMT, Silva SA, Cavenaghi SM, Siqueira Jr JB. Diferenças no padrão de ocorrência da mortalidade neonatal e pós-neonatal no Município de Goiânia, Brasil, 19921996: análise espacial para identificação das áreas de risco. Cad Saude Publica 2001; 17(25):1241-1250.

20. Minuci EG, Almeida MF. Diferenciais intra-urbanos de peso ao nascer no município de São Paulo. Rev Saude Publica 2009; 43(2):256-266.

21. Silva ZP, Almeida MF, Ortiz LP, Alencar GP, Pereira A, Schoeps D, Minuci EG, Novaes HMD. Morte neonatal precoce segundo complexidade hospitalar e rede SUS e não-SUS na Região Metropolitana de São Paulo, Brasil. Cad Saude Publica 2010; 26(1): 123-134.

22. Brasil. Ministério da Saúde (MS). Conselho Nacional de Saúde. Resolução no 196 de 10 de outubro de 1996. Diretrizes e Normas Regulamentadoras de Pesquisas Envolvendo Seres Humanos. Diário Oficial da União 1996; 16 out.

23. Souza GOC. Área de abrangência de atendimento dos hospitais e construção de suas bases territoriais na Região Metropolitana de São Paulo. São Paulo em Perspectiva 2008; 22(2):72-86.

24. Cesar JA, Mano OS, Carlotto K, Gonzales-Chica DA, Mendoza-Sassi RA. Público versus privado: avaliando a assistência à gestação e ao parto no extremo Sul do Brasil. Rev. Bras. Saude Mater. Infant. 2011; 11(3):257-263.

25. Martins CM, Almeida MF. Fecundidade e diferenciais intra-urbanos de desenvolvimento humano, São Paulo, Brasil, 1997. Rev Saude Publica 2001; 35(5):421-427.

26. Brasil. Conselho Nacional de Secretários de Saúde. SUS: avanços e desafios. Conselho Nacional de Secretários de Saúde. Brasília: CONASS; 2006.

27. Leal MC, Campos MR, Cavalini LT, Garbayo LS, Brasil CLP, Szwarcwald CL. Fatores associados à morbi-mortalidade perinatal em uma amostra de maternidades públicas e privadas do Município do Rio de Janeiro, 1999-2001. Cad Saude Publica 2004; 20(1):S20-S33.

28. Cechin J, Martins CB, Leite F. Diferenças assistenciais no pré-natal, parto e puerpério entre as mulheres que utilizaram plano de saúde versus SUS. In: Qualihosp 2009; São Paulo. IX Congresso Internacional de Qualidade em Serviços e Sistemas de Saúde 2009; São Paulo. p. 11-14.

29. Hau LC, Nascimento LFC, Tomazini JE. Geoprocessamento para identificar padrões do perfil de nascimentos na região do Vale do Paraíba. Rev. Bras. Ginecol. Obstet. 2009; 31(4):171-176.

30. Carniel EF, Zanolli ML, Morcillo AM. Fatores de risco para indicação do parto cesáreo em Campinas (SP). Rev. Bras. Ginecol. Obstet. 2007; 29(1):34-40.

31. Barros AJD, Santos IS, Matijasevich A, Domingues MR, Silveira M, Barros FC, Victora CG. Patterns of deliveries in a Brazilian birth cohort: almost universal cesarean sections for the better-off. Rev Saude Publica 2011; 45(4):635-643. 
32. Patah LEM, Malik AM. Modelos de assistência ao parto e taxa de cesárea em diferentes países. Rev Saude Publica 2011; 45(1):185-194.

33. Dias MAB, Domingues RMSM, Pereira APE, Fonseca SC, Gama SGN da, Theme Filha MM, Bittencourt SDA, Rocha PMM, Schilithz AOC, Leal MC. Trajetória das mulheres na definição pelo parto cesáreo: estudo de caso em duas unidades do sistema de saúde suplementar do estado do Rio de Janeiro. Cien Saude Colet 2008; 13(5):1521-1534.

34. Geraldo CF, Garcias GL, Roth, MGM. Prevalência de nascimentos gemelares em Pelotas, Rio Grande do Sul, Brasil. Rev. Bras. Saude Mater. Infant. 2008; 8(4):411-417.

35. Giglio MRP, Lamounier JA, Morais Neto OL de, Cesar CC. Baixo peso ao nascer em coorte de recém-nascidos em Goiânia-Brasil no ano de 2000. Rev. Bras. Ginecol. Obstet. 2005; 27(3):130-136.

Artigo apresentado em 22/10/2012

Aprovado em 01/11/2012

Versão final apresentada em 05/11/2012 